\title{
SAÚDE, AMBIENTE E DESENVOLVIMENTO NAS MICRORREGIÕES DE CHAPECÓ-SC E ERECHIM-RS
}

\section{HEALTH, ENVIRONMENTAL AND DEVELOPMENT IN THE MICRO REGIONS FROM CHAPECÓ-SC AND ERECHIM-RS}

\author{
Jane Kelly Oliveira Friestino \\ Doutora em Saúde Coletiva área Epidemiologia \\ Universidade Federal da Fronteira Sul campus Chapecó \\ jane.friestino@uffs.edu.br \\ Venir Guilherme Baldissera \\ Acadêmico de Medicina, bolsista de Iniciação em Desenvolvimento Tecnológico e Inovação \\ Universidade Federal da Fronteira Sul campus Chapecó \\ venirbaldissera@gmail.com \\ Vitor Hugo Batista Santos \\ Acadêmico de Geografia, estudante voluntário de Iniciação em Desenvolvimento Tecnológico e Inovação \\ Universidade Federal da Fronteira Sul campus Chapecó \\ vitortecnic02000@gmail.com
}

\begin{abstract}
RESUMO
A falta de dados e informações em saúde constitui um dos grandes entraves para o avanço de estudos e pesquisas regionais. Por isso, o objetivo deste estudo foi construir indicadores de saúde e ambiente relacionados aos objetivos da Agenda 2030 nos municípios do Observatório Geográfico da Fronteira Sul. Trata-se de uma pesquisa tecnológica de caráter transversal, realizada nas microrregiões de Chapecó-SC e Erechim-RS. Foram calculados os Índices de Carência Habitacional e os indicadores de saúde: mortalidade infantil, mortalidade geral, mortalidade específica por câncer e suicídio, de todos municípios das duas microrregiões estudadas. $\mathrm{Na}$ análise dos indicadores foram verificadas diferenças entre os coeficientes de mortalidade por neoplasias e mortalidade por lesões autoprovocadas, o que exige intervenções que visem ao desenvolvimento dessas localidades. A identificação dos indicadores sociodemográficos constituiu-se em importante instrumento para a caracterização das particularidades de cada território, facilitando o progresso científico, a gestão, e o aumento da eficiência dos serviços de saúde.
\end{abstract}

Palavras-chave: Indicadores de Saúde. Indicadores Ambientais. Saúde Ambiental. Desenvolvimento Regional. Saúde Coletiva.

\begin{abstract}
The lack of data and information on health constitutes one of the major obstacles to the advancement of regional studies and research. Therefore, this study aim to build indicators of health and environmental, related to the objectives of 2030 Agenda in the cities of the Geographic Observatory of the Southern Border. This is a cross-sectional technological research carried out in microregions of Chapecó-SC and Erechim-RS. The Housing Need Index and the health indicators were calculated, including: infant mortality, general mortality, cancer-specific mortality and suicide of all municipalities of the two microregions studied. In the analysis of the indicators, there were important differences between the coefficients of mortality due to cancer and mortality due to self-inflicted injury, which requires interventions that aimed at the development of these localities. The identification of sociodemographic indicators was an important instrument for characterizing the particularities of each territory, facilitating scientific progress, management and increasing the efficiency of health services.
\end{abstract}

Keywords: Health Indicators. Environmental Indicators. Environmental Health. Regional Development. Collective Health.

Recebido em: 22/07/2019

Aceito para publicação em: 24/04/2020. 


\section{INTRODUÇÃO}

A promoção da saúde ocorre por meio de um complexo processo condicionado por diferentes fatores, incluindo o ambiente. Por meio dela busca-se melhorar a condição de vida das pessoas, incluindo os determinantes sociais e de saúde como uma engrenagem fundamental para a conscientização sobre a necessidade de se ter um desenvolvimento sustentável, garantindo assim a saúde da população (CZERESNIA; RIBEIRO, 2000).

Para incrementar o desenvolvimento sustentável, as Nações Unidas pactuaram, em 2015, a proposta da Agenda 2030 (UNITED NATIONS, 2015). Sua utilização baseia-se em informações presentes nas realidades locais, valorizando as potencialidades e saberes existentes, em uma perspectiva que visa ao atendimento das demandas particulares, sem deixar que os sujeitos sejam prejudicados pelo desconhecimento dessas informações. Assim, a Agenda 2030 constitui-se em um importante recurso norteador de ações, definindo 17 objetivos e 169 metas para um mundo melhor. Vale ressaltar que são abordadas neste artigo os indicadores referentes aos seguintes objetivos: 3) Saúde e Bem-estar, 6) Água Potável e Saneamento e 11) Cidades e comunidades saudáveis² (UNITED NATIONS, 2015).

É válido destacar que a promoção da saúde elucida uma nova forma de cuidado, que reconhece os determinantes relativos ao ambiente como sendo fundamentais às condições de vida das populações, pois supõe que, se compreendermos bem as causas e as consequências dos problemas em saúde, aumentam as chances de se realizar uma ação assertiva (PAIM; ALMEIDA FILHO, 2014). Vale lembrar que quando se fala em problemas de saúde, incluem-se os seguintes conceitos: "danos (prejuízos), mortes, doenças, agravos, sequelas, riscos, carências e vulnerabilidades". É importante lembrar que os três últimos conceitos se referem ao estado de saúde de uma população (PAIM; ALMEIDA FILHO, 2014).

De acordo com essa perspectiva, as desigualdades sociais, econômicas e tecnológicas impactam diretamente a deterioração socioambiental (HACON; SCHUTZ; BERMEJO, 2005). Nessa vertente, a Vigilância em Saúde emerge como um modelo de assistência que tem por finalidade a implementação do Sistema Único de Saúde (SUS) no Brasil e que compreende a saúde como um produto social, derivado das relações sociais presentes em um dado cenário político, econômico, ideológico e cultural. Os debates sobre Vigilância da Saúde incluem a análise de situações de saúde, que toma como objetos as situações de saúde dos distintos grupos populacionais, em função de suas condições de vida (SANTIAGO; et al, 2008).

Com isso, é possível entender que a concepção de "natureza" não se dissocia da concepção de "sociedade" e os múltiplos saberes podem ser contemplados na formação médica, a partir de um olhar voltado ao desenvolvimento científico e tecnológico pautado na ecologia dos saberes, que una o pensamento à técnica (SANTANA, 2016). Essa proposta de aproximação culminou na execução desta pesquisa, que - embora faça parte do campo da Saúde Coletiva - possui uma interlocução interdisciplinar e transdisciplinar, que objetiva contribuir para o expressivo entendimento das dinâmicas presentes na condição de vida dos sujeitos, por meio de uma reflexão constante sobre as bases filosófico-epistemológicas da ciência geográfica.

A organização das informações é papel da Vigilância em Saúde e agregar o espaço geográfico tem sido ato valioso, conforme Guimarães (2016). Esse gesto permite o uso dessas informações agregadas ao espaço geográfico no contexto do estudo das doenças, com uma abordagem capaz de analisar a saúde como uma forma mais objetiva, possibilitando uma compreensão aprofundada na relação estabelecida entre o espaço, as doenças e os agravos à saúde (CZERESNIA; RIBEIRO, 2000).

Assim, é fundamental pautar-se no referencial da Vigilância em Saúde, entendendo-a como um processo contínuo e sistemático de coleta, consolidação, análise e disseminação de dados sobre eventos relacionados à saúde, visando o planejamento e a implementação de medidas de Saúde Pública para a proteção da saúde da população, a prevenção e controle de riscos, agravos e doenças, bem como para a promoção da saúde nos territórios onde as pessoas vivem, tornando-os mais saudáveis (BRASIL, 2013).

Na Universidade Federal da Fronteira Sul, o Núcleo de Estudos Território, Ambiente e Paisagem (NETAP), desde a sua concepção, busca se tornar uma referência nos estudos geográficos da região de abrangência e influência da Universidade e seu entorno, promovendo análises e estudos em relação ao desenvolvimento territorial e ambiental, na dinâmica e no comportamento dos elementos

2 Plataforma Agenda 2030: http://www.agenda2030.com.br/

Hygeia

v.16

p.25 - 36,

2020

página 26 
naturais da paisagem, nas relações entre os espaços urbano e rural. Os objetivos são: manter uma reflexão constante sobre as bases filosófico-epistemológicas da ciência geográfica, além de promover o contínuo diálogo da pesquisa com o aperfeiçoamento das técnicas e estratégias de ensino da geografia. Para alcançar os objetivos do NETAP, foi instituído o projeto de pesquisa "Observatório Geográfico da Fronteira Sul: construindo e compartilhando experiências para a democratização do acesso às informações regionais" financiado pela FAPESC, com atenção voltada às microrregiões dos municípios de Chapecó-SC e Erechim-RS. O referido projeto de pesquisa tornou-se estratégico por se constituir em referência nos estudos geográficos, na área de abrangência da região fronteira sul do Brasil.

No âmbito do "Observatório Geográfico da Fronteira Sul", foi executado o projeto de iniciação em desenvolvimento tecnológico e inovação intitulado: "Informações em Saúde no Observatório Geográfico da Fronteira Sul", que teve como objetivo de enriquecer a base de dados do referido Observatório com informações sobre os principais indicadores de saúde nos municípios das microrregiões de abrangência do projeto e sobre a definição da localização e estruturação da rede pública de atendimento de saúde dos municípios de Chapecó-SC e Erechim-RS, nos diferentes níveis de complexidade.

O referido projeto fundamentou-se numa compreensão do território como um constructo de agenciamentos de componentes de ordem biológica, social, imaginária, semiótica, afetiva, política e cultural. Trata-se de um conceito complexo que deve ser abordado na perspectiva de libertar o conhecimento local oriundo das necessidades e realidades locais (LIMA; YASUI, 2014) no sentido de balizar as estratégias de promoção de saúde, prevenção e controle de doenças.

Após breve apresentação dos pressupostos que balizam a presente pesquisa, seguem algumas características das áreas de estudo. A microrregião de Erechim é composta por 30 municípios, que no ano de 2015 apresentou um somatório de 219.107 habitantes (IBGE, 2017). Localiza-se ao Norte do estado do Rio Grande do Sul e tem uma economia constituída majoritariamente por pequenas propriedades rurais, que produzem especialmente milho, soja e trigo. Há também a criação de animais, que formam a agroindústria regional, baseada na carne suína e de frango, e na produção de leite (PESSÔA; RUCKERT; RAMIRES, 2017).

A microrregião de Chapecó localiza-se no Oeste do estado de Santa Catarina. É composta por 38 municípios que possuem uma população total de 434.575 habitantes (ano-base: 2015) (IBGE, 2017). A predominância migratória e cultural da microrregião foi estabelecida por colonos italianos, alemães e poloneses, que chegaram na região em meados do século $X X$, oriundos principalmente do Rio Grande do Sul. A economia é muito ligada ao agronegócio; a região é uma grande exportadora de carne de aves e suínos (BALDANÇA, 2008). No que diz respeito à saúde - embora existam diferenças entre os municípios da microrregião - ela é considerada eficiente em relação ao uso de recursos destinados à Saúde Pública (KAVESKI; MAZZIONI; HEIN, 2013).

Ao se pensar em informações sobre saúde em nosso país, há que se referir ao Sistema Único de Saúde (SUS), no qual temos a territorialização como uma das bases de organização e estruturação dos serviços e práticas de atenção à saúde. Isso porque a vinculação entre a geografia e a saúde permite conhecer a realidade da população de determinado território, fazendo com que as práticas adotadas sejam voltadas para as características epidemiológicas, políticas, sociais e culturais particulares de cada localidade. Essa vinculação favorece o atendimento dessas características particulares e fortalece o vínculo entre equipe e pacientes (MONKEN; BARCELLOS, 2005).

Atualmente, a análise de saúde ambiental é considerada um fator indispensável para o controle social, para definir as necessidades e subsidiar a formulação de políticas públicas (HACON; SCHUTZ; BERMEJO, 2005; BARCELLOS, 2006; CANAVESE; POLIDORO, 2013). Vale enfatizar, entretanto, que há debilidade no monitoramento desse tema nas áreas de estudo. Justamente por isso, é preemente a investigação dos avanços e retrocessos, para que se possa subsidiar a definição de prioridades na agenda regional.

Como parte desse contexto global entende-se a importância de se estudar conjuntamente os temas saúde, ambiente e desenvolvimento, com o intuito de ofertar um suporte para a implementação de ações em busca da melhoria da qualidade de vida e de saúde das populações.

O objetivo deste estudo foi apresentar uma seleção sucinta dos resultados de indicadores em saúde e ambiente relacionados aos objetivos da Agenda 2030, disponíveis para consulta pública. Buscou-se construir indicadores de saúde, por meio de diferentes coeficientes de mortalidade dos municípios pertencentes às microrregiões de Chapecó-SC e Erechim-RS, de forma a permitir a visualização de 
suas particularidades, possibilitando a identificação de problemas e desenvolvimento de estratégias de enfrentamento.

\section{MATERIAL E MÉTODOS}

Trata-se de uma pesquisa tecnológica de caráter transversal, realizada nas microrregiões de Chapecó-SC e Erechim-RS. Este estudo faz parte de um projeto maior, intitulado: "Observatório geográfico da fronteira sul: construindo e compartilhando experiências para democratização do acesso às informações regionais". A inclusão do tema "Saúde" no Observatório surgiu a partir de uma reunião geral de organização do trabalho e formação de um conselho do projeto com a definição de temas prioritários para encaminhamento da pesquisa, precedido de realização de trabalhos de campo e composição de parcerias. Foram coletados os seguintes indicadores: taxa de mortalidade infantil, taxa de mortalidade geral, taxa de mortalidade específica por câncer e suicídio, taxa de fecundidade, além da taxa de mortalidade proporcional por idade, para as microrregiões de Chapecó-SC e Erechim-RS,

Os dados obtidos foram organizados em um banco de dados, produzido por meio do Planilhas on line do Google. Posteriormente, foram analisadas no Excel. A sistematização ocorreu por meio da construção de tabelas, gráficos e mapas. Depois de ter sido realizada a concentração das informações, as mesmas foram submetidas a comparações e cruzamentos.

A partir disso, foram realizadas estatísticas descritivas, retratando todos os temas abordados na proposta. Intentou-se realizar o mapeamento dos indicadores de acordo com a área de abrangência dos Centros de Saúde da Família (CSF) das duas cidades polo das microrregiões estudadas. Porém, após a realização de consulta junto ao órgão municipal responsável pelo mapeamento digital das unidades, notou-se que estes encontravam-se desatualizados, inviabilizando a ideia inicial que seria a produção de mapas temáticos dos indicadores.

A presente pesquisa utilizou dados de domínio público disponibilizados pelo Departamento de Informática do SUS (DATASUS), o qual exibe dados agregados, não apresentando informações de condição de saúde individual, tampouco houve contato com informações a respeito de questões que envolvessem a identificação de sujeitos. Sendo assim, conforme a Resolução 466 de 2012 do Conselho Nacional de Saúde, não necessitou de apreciação ética.

\section{Índice de carência habitacional}

A partir dos dados disponibilizados pela plataforma de dados "Atlas do Desenvolvimento Humano no Brasil" (AtlasBrasil) e com a metodologia proposta por Canavese e Polidoro (2013) calculouse um indicador baseado nos dados de abastecimento de água, esgotamento sanitário e destinação do lixo, denominado de Índice de Carência Habitacional (ICH). Os pontos variáveis que compõem o índice são, portanto: o abastecimento de água, o tipo de instalação sanitária e a regularidade da coleta de lixo e destino.

A determinação desse coeficiente é feita para que se possa dimensionar as dificuldades de habitação em cada município. Vale ressaltar que essa determinação não tem o intuito de organizar os dados como um ranking de municípios. É importante também frisar que o ICH não é disponibilizado por outra plataforma de armazenamento de dados para os municípios analisados e que utilizada uma metodologia para determinar os resultados em um intervalo entre 0 (valor correspondente aos municípios mais carentes) a 1 (valor correspondente aos municípios menos carentes).

Para calcular o valor, é necessário utilizar o valor de um dos três dados coletados e subtrair uma centena. Logo depois, dividimos o resultado pelo número negativo de cem. Na composição final, cada um dos dados apresentados possui um peso: $\mathrm{ICH}-$ Abastecimento de água (Peso 3); $\mathrm{ICH}-$ Tipo de instalação sanitária (Peso 2); ICH - Regularidade da coleta de lixo e destino (Peso 1). $\mathrm{O}$ valor final é calculado por meio de uma média ponderada, segundo Canavese e Polidoro (2013). 


$$
\text { Îndice }(\mathrm{ICH})=\frac{\text { Valor Observado }-100}{0-100}
$$

\begin{tabular}{|c|c|}
\hline ICH - água & Entra com peso 3 na composição do ICH final \\
\hline ICH - lixo & Entra com peso 1 na composição do ICH final \\
\hline ICH - instalação sanitária & Entra com peso 2 na composiça do ICH final \\
\hline \multicolumn{2}{|c|}{ Fonte: Canavese e Polidoro (2013). }
\end{tabular}

Quanto às variáveis que compõem a distribuição de pesos que compõem o coeficiente de cada um dos dados estruturais do ICH propostas pelo IPPUR (2003), notamos que não é disponibilizada a descrição dos tipos de serviços efetuados por cada residência em cada município em plataformas de dados. Ou seja, coletamos somente um número de residências que têm ou não acesso aos serviços para a formulação. Para hierarquizar o $\mathrm{ICH}$ foram estabelecidas três categorias: extremo $\mathrm{ICH}$ de 0 até 0,5 ; alto ICH de 0,5 até 0,8 e baixo ICH de 0,8 até 1 (CANAVESE; POLIDORO, 2013).

\section{Indicadores de saúde}

Entendendo o contexto regional utilizou-se a plataforma DATASUS, por meio do Sistema de Informação de Mortalidade (SIM), no qual foram coletados dados de mortalidade geral, mortalidade por neoplasias e mortalidade por lesão autoprovocada. Os referidos dados foram divididos por faixas etárias. Além disso, também foram coletados dados referentes ao número de nascidos vivos nos 30 municípios que compõem a microrregião de Erechim-RS e nos 38 municípios da microrregião de Chapecó-SC.

Para tanto, foram considerados o período de 2000 a 2015, compondo uma série histórica de 16 anos (2000 a 2015). Foi realizado o levantamento nos 38 municípios da microrregião de Chapecó-SC, que compreende: Águas de Chapecó, Águas Frias, Bom Jesus do Oeste, Caibi, Campo Erê, Caxambu do Sul, Chapecó, Cordilheira Alta, Coronel Freitas, Cunha Porã, Cunhataí, Flor do Sertão, Formosa do Sul, Guatambú, Iraceminha, Irati, Jardinópolis, Maravilha, Modelo, Nova Erechim, Nova Itaberaba, Novo Horizonte, Palmitos, Pinhalzinho, Planalto Alegre, Quilombo, Saltinho, Santa Terezinha do Progresso, Santiago do Sul, São Bernardino, São Carlos, São Lourenço do Oeste, São Miguel da Boa Vista, Saudades, Serra Alta, Sul Brasil, Tigrinhos e União do Oeste. O levantamento foi realizado também nos 30 municípios da microrregião de Erechim: Aratiba, Áurea, Barão de Cotegipe, Barra do Rio Azul, Benjamin Constant do Sul, Campinas do Sul, Carlos Gomes, Centenário, Cruzaltense, Entre Rios do Sul, Erebango, Erechim, Erval Grande, Estação, Faxinalzinho, Floriano Peixoto, Gaurama, Getúlio Vargas, Ipiranga do Sul, Itatiba do Sul, Jacutinga, Marcelino Ramos, Mariano Moro, Paulo Bento, Ponte Preta, Quatro Irmãos. São Valentim, Severiano de Almeida, Três Arroios, Viadutos.

Para a coleta de dados referentes aos óbitos por neoplasias foram selecionados os códigos C00 a C97 do Capítulo II - Neoplasias [tumores], da $10^{\text {a }}$ Revisão da Classificação Internacional de Doenças (CID-10) e Capítulo XIX - Lesões, autoprovocadas intencionalmente, sendo utilizados os códigos X70-X84, caracterizando óbitos em decorrência de suicídio (RIPSA, 2008).

Após o levantamento dos óbitos no período, foram calculados os indicadores segundo RIPSA (2008): coeficiente médio de mortalidade geral, taxa de mortalidade infantil, coeficiente médio de mortalidade por neoplasias, proporção de óbitos por neoplasia, coeficiente médio de mortalidade por lesão autoprovocada, proporção de óbitos por lesão autoprovocada, possibilitando assim, a representação gráfica da ocorrência nos municípios estudados. Optou-se por estudar essas duas causas de óbito, pois a própria literatura aponta como uma problemática vivenciada pelo oeste catarinense, assim como no norte e noroeste do Rio Grande do Sul (HESS, 2017; KUIAVA et al, 2018).

Vale lembrar que a taxa de mortalidade infantil é calculada como o número de óbitos de crianças menores de um ano, dividido por mil nascidos vivos, na população residente em determinado local, em um período de tempo considerado (RIPSA, 2008). Neste estudo foi feita a média de todos os municípios das microrregiões de Chapecó e Erechim e comparou-se com a taxa de mortalidade infantil dos municípios de Chapecó e Erechim, pois esses são os únicos municípios das duas regiões que possuem mais de 100 mil habitantes, conforme orienta a Rede Integrada de Informações para a Saúde (RIPSA). Tal configuração justifica-se pela grande quantidade de pequenos municípios nas microrregiões estudadas, que não possuem número de ocorrências suficiente para que o cálculo seja realizado individualmente. 


\section{RESULTADOS E DISCUSSÃO}

De acordo com o Censo de 2000, a microrregião de Chapecó-SC possuía uma população total de 405.123 habitantes, enquanto que em Erechim-RS, nesse mesmo período, a população era de 211.685 habitantes.

Na microrregião de Chapecó foram estudados 38 municípios. Dentre os resultados obtidos com o cálculo de $\mathrm{ICH}$, é relevante apontar que 36 (94,7\%) municípios encontram-se com baixo $\mathrm{ICH}$, destacando-se o município de Nova Erechim $(\mathrm{ICH}=0,985)$ que demonstra possuir bons serviços básicos de saneamento, coleta de lixo e fornecimento de água. Ainda na microrregião de Chapecó, os municípios Cordilheira Alta $(\mathrm{ICH}=0,769)$ e São Bernardino $(\mathrm{ICH}=0,786)$, apresentaram alto $\mathrm{ICH}$ respectivamente. Uma realidade semelhante foi percebida na microrregião de Erechim-RS, em que foram analisados 30 municípios, sendo que, 29 municípios apresentaram baixo $\mathrm{ICH}$, e somente o município de Viadutos foi classificado como alto $\mathrm{ICH}(0,744)$. Ambas regiões não apresentaram municípios com extremo ICH, conforme pode se observar na tendência apresentada na Figura 1.

Figura 1 - Índice de Carência Habitacional nos municípios das microrregiões de Chapecó e Erechim

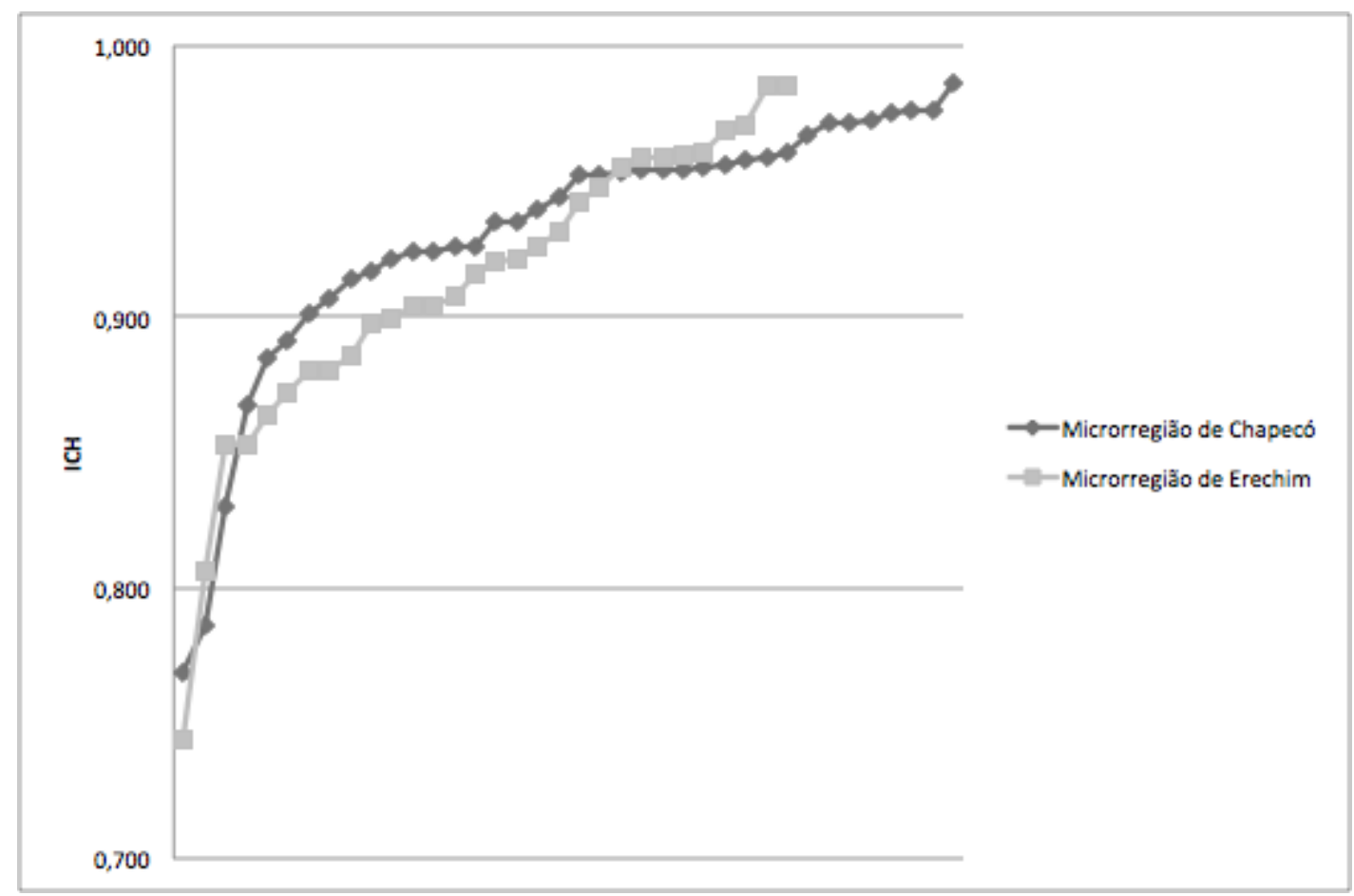

Fonte: Atlas Brasil. Elaborado pelos autores

A utilização do ICH mostra-se relevante pela produção e integração dos dados. Seu cruzamento de informações possibilita uma melhor análise e viabiliza a construção cartográfica para visualização.

Esses dados se conectam ao $6^{\circ}$ (sexto) item da Agenda 2030: "Assegurar a disponibilidade e gestão sustentável da água e saneamento para todos" que tem como objetivo alcançar o acesso universal e, de modo equitativo, à água potável e ao saneamento e higiene de forma adequada. Isso possibilitaria uma gestão hídrica adequada em todos os níveis e aumentaria a eficiência da utilização desses recursos. O tema é um dos dados estruturais utilizados para a determinação do $\mathrm{ICH}$. Desse modo, podemos dimensionar as dificuldades existentes em relação ao acesso à água e ao saneamento nas microrregiões de Chapecó e Erechim, facilitando a análise de pontos de melhoria urbana (UNITED NATIONS, 2015).

De acordo com Polidoro, Takeda e Barros (2009), a utilização de indicadores, como o "Índice de Carência Habitacional", mostra-se altamente viável em estudos que envolvem questões de saneamento básico a partir dos dados do Censo IBGE. Eles possibilitam realizar avaliações acerca da condição de saúde da população. Permitem também o cruzamento de informações que possuem 
uma ligação direta entre elas, além de proporcionar ferramentas para a formulação de políticas públicas voltadas às questões do meio ambiente.

Não obstante, também é visível a aproximação dos municípios com maior ICH da concretização de parte do $11^{\circ}$ (décimo primeiro) item da Agenda 2030: "Tornar as cidades e os assentamentos humanos inclusivos, seguros, resilientes e sustentáveis" visa garantir o acesso de todos a uma habitação segura, a preço acessível e com serviços básicos. Possibilita também o acesso a locais públicos inclusivos e verdes. O tema também trata de (re)inclusão para mulheres e crianças, idosos e pessoas com necessidades especiais. Assegura o cumprimento do papel de uma sociedade que não delimite requisitos para utilização de seus bens de serviços. Fomenta a difusão de informações importantes e de seu desenvolvimento sustentável e estilos de vida que sigam em harmonia com a natureza (UNITED NATIONS, 2015).

Apesar das limitações dos dados disponíveis, e da dificuldade pela não definição de critérios específicos para mensurar o progresso dos municípios nos dois objetivos da Agenda 2030 supracitados, os resultados observados no cálculo do $\mathrm{ICH}$ da grande maioria dos municípios estudados nas duas microrregiões indicam adequada oferta de serviços públicos essenciais, o que se aproxima do proposto pelos objetivos.

Quanto ao o terceiro objetivo da Agenda 2030: "Assegurar uma vida saudável e promover o bemestar para todos, em todas as idades" e tem entre suas metas a de reduzir a mortalidade neonatal para pelo menos 12 por mil nascidos-vivos, bem como a de reduzir a mortalidade de crianças menores de 5 anos para pelo menos 25 por mil, além de garantir a cobertura universal de saúde, com acesso a medicamentos e vacinas essenciais de modo seguro, eficiente e de qualidade, a um valor acessível a toda a população (UNITED NATIONS, 2015).

Embora as metas designadas pela Agenda 2030 utilizem a mortalidade neonatal, os dados disponíveis se referem a taxa de mortalidade infantil, que é usualmente classificada como alta quando superior a 50 por mil; considerada média quando apresenta valores entre $20 \%$ e $49 \%$ e baixa quando inferior a $20 \%$ (RIPSA, 2008). Nesse sentido, observou-se no município de Chapecó uma taxa de mortalidade infantil de $12,6 \%$, enquanto que em sua microrregião a taxa foi de $13,2 \%$. Já no município de Erechim a taxa foi de $13,6 \%$, enquanto que na sua respectiva microrregião observou-se a taxa de $11,6 \%$. Apesar da pequena variação, todos os valores observados para o coeficiente de mortalidade infantil são considerados baixos e se adequam a meta da Agenda 2030 de reduzir a mortalidade na infância.

As baixas taxas de mortalidade infantil na microrregião de Chapecó são, provavelmente reflexos pelo menos em parte - dos melhores indicadores de saneamento presentes na microrregião. No entanto, vale ressaltar que esse indicador deve ser utilizado com cautela quando se trata de municípios com populações inferiores a 100 mil habitantes, pois o impacto dos óbitos na realização dos cálculos pode causar uma falha na interpretação da realidade.

Outra ocorrência de impacto no desenvolvimento regional é a presença de casos de câncer na população, pois além de necessitar de uma rede de serviço de saúde de alta complexidade para os casos existente, sua ocorrência pode ser minimizada quando existem políticas de promoção da saúde e acesso aos serviços de prevenção aos agravos. A ocorrência de câncer é atualmente um problema de saúde pública comum às duas microrregiões estudadas (HESS, 2017; KUIAVA et al, 2018). O potencial carcinogênico da exposição solar, da exposição aos agrotóxicos, somados ao estilo de vida sedentário e com pouca diversidade de alimentação, além de uma expectativa de vida elevada da população, favorecem o desenvolvimento de doenças crônicas não transmissíveis, como é o caso do câncer. Com isso, identificou-se que os cálculos de coeficiente de mortalidade por neoplasias somam para a microrregião de Chapecó um acúmulo importante nas taxas de mortalidade: perfazem no município de Palmitos 367,5 óbitos por cada 100.000 habitantes, no período estudado, e na microrregião de Erechim, o município de Campinas do Sul, com uma perspectiva de 179 óbitos por neoplasias a cada 100.000 habitantes, e além disso, todos os municípios desta microrregião apresentaram coeficientes médios de mortalidade por neoplasia maiores que 100 por 100.000 habitantes, conforme Figura 2. 
Figura 2 - Coeficiente médio de mortalidade por neoplasias de residentes dos municípios da microrregião de Chapecó (A) e Erechim (B), nos anos de 2000, 2007, 2009, 2010 e 2015
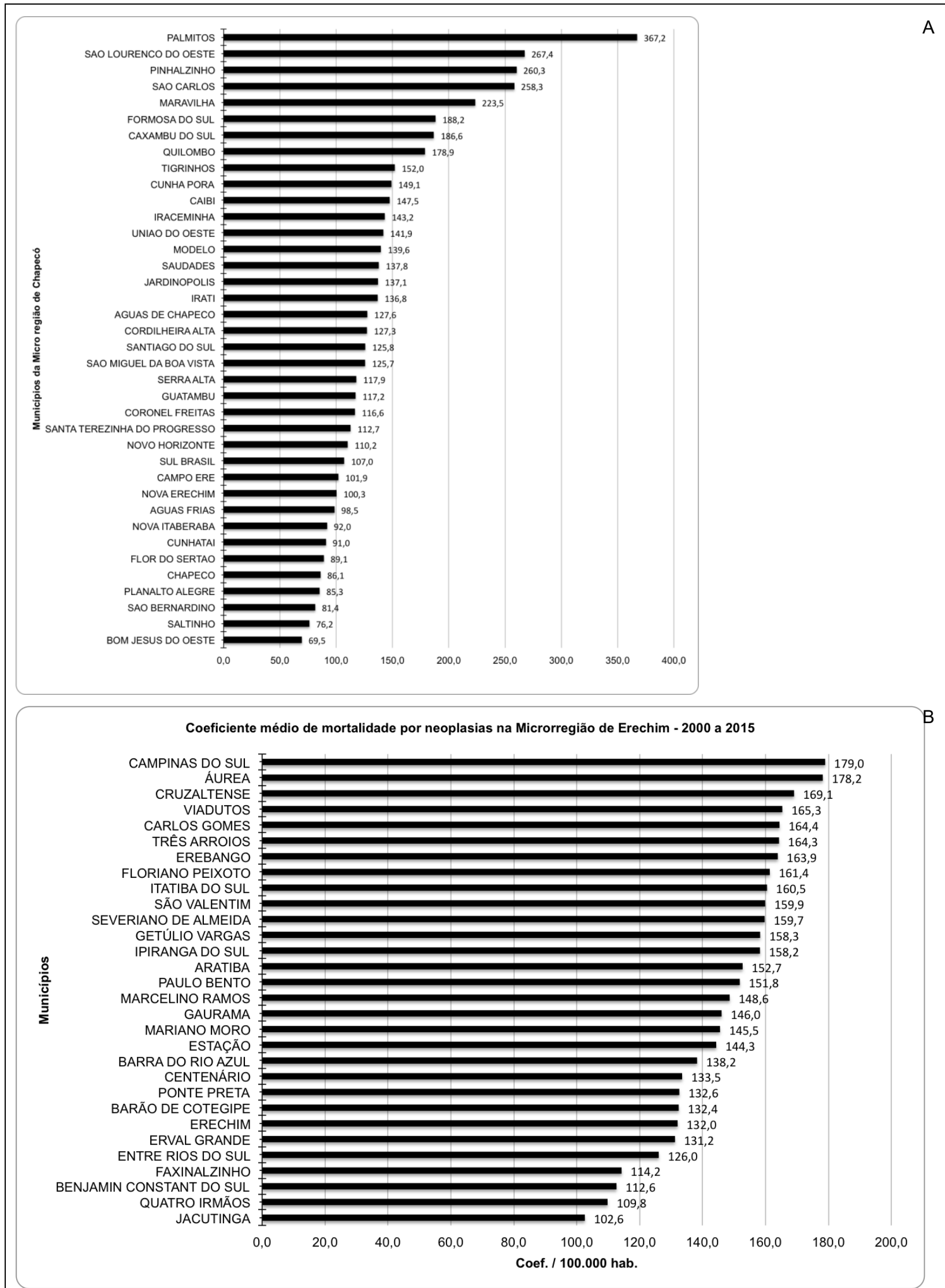

Fonte: Datasus. Elaborado pelos autores 
Outro indicador importante é o acompanhamento das ocorrências de óbito por suicídio, também apontado como uma problemática comum para as microrregiões. Meneghel e Moura (2018) trazem como um dos possíveis determinantes do suicídio a influência germânica, presente na maioria dos municípios estudados, em geral, pode-se associar aos possíveis determinantes culturais e sociais, além das questões orgânicas envolvidas no processo. Além disso, diversas pesquisas estão sendo desenvolvidas para investigar a presença de comportamento suicida em agricultores expostos à agrotóxicos, pois pressupõe-se que exista relação entre a exposição aos mesmos e o desenvolvimento de transtornos mentais, como também uma incidência maior de óbitos por lesões auto provocadas na região sul do Brasil em comparação com as demais regiões brasileiras (SOUZA, COSTA, RAMOS, 2016; MOTA, 2015).

Em relação aos óbitos por lesões auto provocadas nas microrregiões, os resultados da análise dos indicadores de mortalidade demonstraram que no município de Novo Horizonte, pertencente à microrregião de Chapecó-SC, 12,8\% dos óbitos registrados correspondem ao suicídio, enquanto que na microrregião de Erechim-RS, o município que apresentou a maior proporção foi Cruzaltense, com $3,4 \%$ dos óbitos atribuídos a essa causa.

Figura 3 - Proporção de óbitos por lesão autoinfligida de residentes dos municípios da microrregião de Chapecó (A) e Erechim (B), nos anos de 2000, 2007, 2009, 2010 e 2015

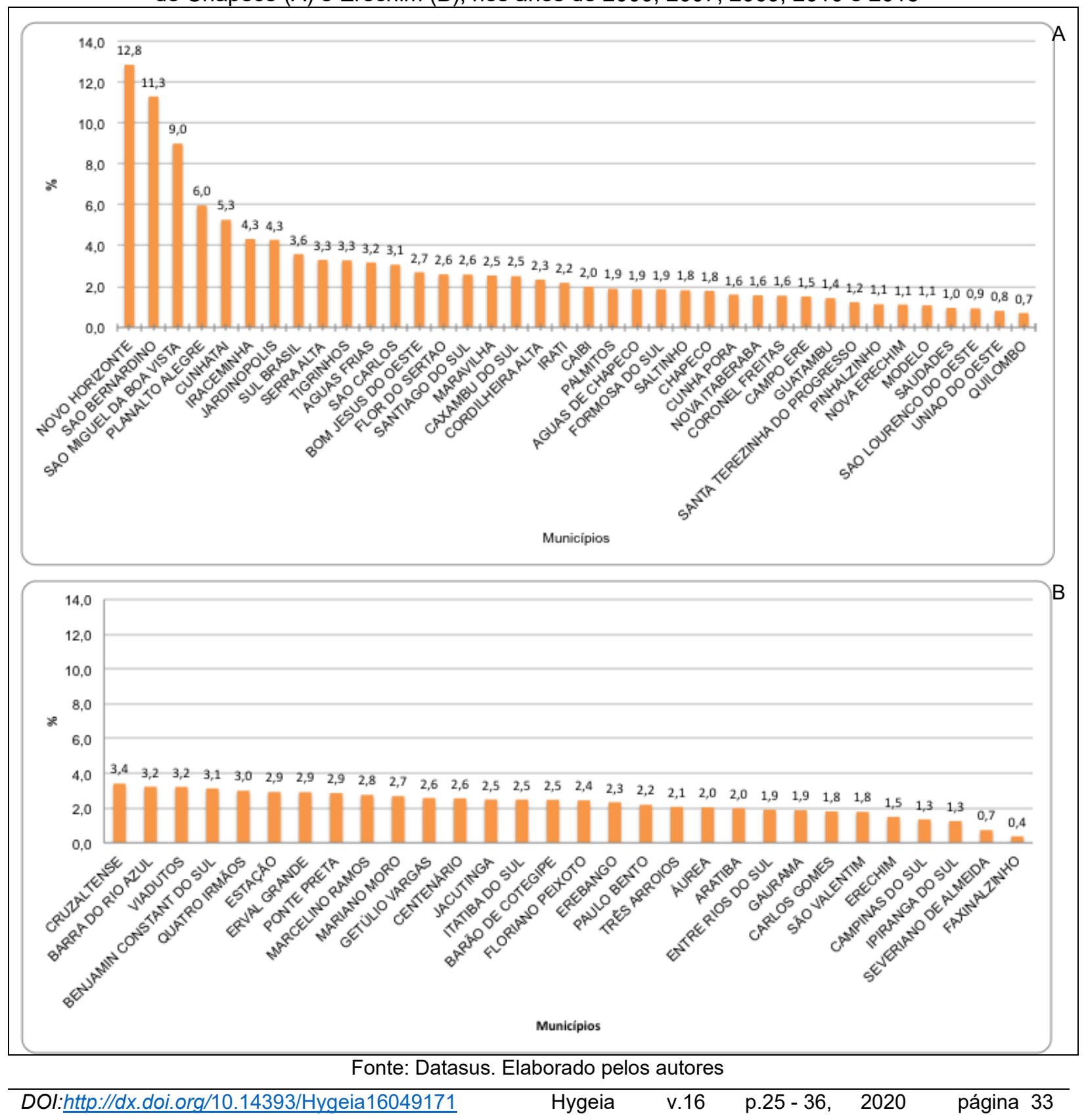


Além disso, devem ser consideradas as questões econômicas que estão diretamente ligadas à realidade da população local: as dificuldades de sobrevivência, a entrada do capitalismo no campo, a expansão do assalariamento rural o empobrecimento e a perda de autonomia seriam fatores de riscos e, por vezes, condicionantes para que o óbito por suicídio aconteça (MENEGHEL; MOURA, 2018).

Sendo assim, o (re)conhecimento da região Fronteira Sul e suas interfaces com o desenvolvimento regional foram apresentados por meio de informações em saúde no Observatório Geográfico da Fronteira Sul, os quais encontram-se disponíveis no seguinte endereço eletrônico: https://observatoriogeouffs.wordpress.com que é o local previsto para divulgação de todas as ações do grupo de pesquisa. Dentre os indicadores disponibilizados na plataforma estão: planejamento urbano, infraestrutura urbana, dados de população, economia e meio ambiente; também como pirâmides etárias e outras informações ligadas a saúde.

Por tratar-se de um estudo que utilizou dados secundários, uma de suas limitações reside no fato de que os registros podem possuir erros ou ainda serem subnotificados, o que dificulta assim a interpretação das ocorrências listadas.

\section{CONCLUSÃO}

A disponibilização de informações em saúde - de forma rápida e com abrangência regional contribui com os princípios e diretrizes do Sistema Único de Saúde e promove o empoderamento do poder popular por meio da socialização do saber, feita de forma mais clara e ampla. Além disso, vale pontuar sua contribuição à formação de acadêmicos de diferentes cursos de graduação, tais como: medicina, geografia e engenharia ambiental e sanitária. A presente pesquisa realizou um recorte analítico à luz do campo da Saúde Coletiva, reconhecendo a necessidade de uma abordagem interdisciplinar e transdisciplinar voltada à complexidade inerente às questões de desenvolvimento e saúde. O desenvolvimento do estudo fomentou o debate, que resultou numa compreensão mais aprofundada das regiões em que habitamos e das formas como essas regiões podem ser analisadas, além de ampliar a percepção dos circuitos espaciais que envolvem as microrregiões observadas.

Em geral, as duas microrregiões estudadas possuem semelhanças entre si, contudo existem particularidades em seus indicadores municipais. $\mathrm{Na}$ análise, foram verificadas diferenças importantes entre os coeficientes de mortalidade por neoplasias e mortalidade por lesões autoprovocadas, o que exige intervenções que visem o desenvolvimento dessas localidades, como também apontam a necessidade em se fortalecer as redes de atenção à saúde destas localidades, sendo primordial existirem estratégias de prevenção à esses agravos.

De um modo geral, todos ainda estamos em busca da concretização dos objetivos da Agenda 2030, para isso, o uso da metodologia adotada neste estudo possibilitou a vigilância em saúde dos municípios das microrregiões de Chapecó e Erechim. Por tratar-se de dados de domínio público, o método utilizado pode ser facilmente aplicado para outras localidades, a fim de subsidiar ações para que de fato os objetivos de desenvolvimento sustentável, pactuado para essa década, possam se concretizar.

\section{REFERÊNCIAS}

BALDANÇA, Karina Barbosa Poffo et al. Paralelo do desenvolvimento socioeconômico das microrregiões de Araranguá, Chapecó, Florianópolis e Tijucas. 2008. Trabalho de Conclusão de Curso (Graduação em Ciências Econômicas) - Universidade Federal de Santa Catarina, Florianópolis, 2008. Disponível em: https://repositorio.ufsc.br/xmlui/handle/123456789/122436. Acesso em: 09 jun. 2019.

BARCELLOS, Christovam; QUITÉRIO, Luiz Antônio Dias. Vigilância ambiental em saúde e sua implantação no Sistema Único de Saúde. Revista de Saúde pública, v. 40, p. 170-177, 2006. https://doi.org/10.1590/S0034-89102006000100025

CANAVESE, Daniel; POLIDORO, Mauricio. Uma análise integrada de saúde e ambiente e o desenvolvimento do Litoral do Paraná. Hygeia: Revista Brasileira de Geografia Médica e da Saúde, v. 9, n. 17, 2013. 
CZERESNIA, Dina; RIBEIRO, Adriana Maria. O conceito de espaço em epidemiologia: uma interpretação histórica e epistemológica. Cadernos de Saúde Pública, v. 16, p. 595-605, 2000. https://doi.org/10.1590/S0102-311X2000000300002

GUIMARÃES, Raul Borges. Geografia e saúde coletiva no Brasil. Saúde e sociedade, v. 25, p. 869879, 2016. https://doi.org/10.1590/s0104-12902016167769

HACON, Sandra; SCHUTZ, Gabriel; BERMEJO, Pedro Más. Indicadores de saúde ambiental: uma ferramenta para a gestão integrada de saúde e ambiente. Cadernos de Saúde Coletiva, v. 13, n. 1, p. 45-66, 2005.

HESS, Sonia. A mortalidade por causas violentas no Estado e nas Microrregiões de Santa Catarina. Revista Encontros Teológicos, v. 32, n. 3, p. 429-450, 2017.

IBGE. Instituto Brasileiro de Geografia e Estatística. Cidades. 2017. Disponível em: https://cidades.ibge.gov.br/. Acesso em: 29 maio 2019.

IPPUR. Instituto de Pesquisa e Planejamento Urbano e Regional. Índice de Carência Habitacional Nota Metodológica. Observatório da Metrópole. Universidade Federal do Rio de Janeiro, 2003.

KAVESKI, Itzhak David Simão; MAZZIONI, Sady; HEIN, Nelson. A eficiência na utilização de recursos no setor de saúde: uma análise dos municípios do Oeste Catarinense. Revista de Gestão em Sistemas de Saúde, v. 2, n. 2, p. 53-84, 2013. ISSN 2316-3712. Disponível em: http://www.revistargss.org.br/ojs/index.php/rgss/article/view/72. Acesso em: 30 maio 2019. https://doi.org/10.5585/rgss.v2i2.72

KUIAVA, Victor Antonio, et al. Epidemiological profile of esophageal cancer mortality in Rio Grande do Sul and its health Regions. Clinical \& Biomedical Research, v. 38, n. 3, p. 213-217, 2018. https://doi.org/10.4322/2357-9730.84096

LIMA, Elizabeth Maria Freire de Araújo; YASUI, Silvio. Territórios e sentidos: espaço, cultura, subjetividade e cuidado na atenção psicossocial. Saúde em debate, v. 38, p. 593-606, 2014. https://doi.org/10.5935/0103-1104.20140055

MONKEN, Maurício; BARCELLOS, Christovam. Health surveillance and territory: theoretical and methodological possibilities. Cadernos de saúde pública, v. 21, n. 3, p. 898-906, 2005. https://doi.org/10.1590/S0102-311X2005000300024

MOTA, Adeir Archanjo da. Cartografia do Suicídio no Brasil no período de 1979-2011. Hygeia Revista Brasileira de Geografia Médica e da Saúde, v. 11, n. 20, p. 85 - 98, 27 jul. 2015.

UNITED NATIONS. Transforming our world: the 2030 Agenda for Sustainable Development. 2015. Disponível em: https://sustainabledevelopment.un.org/post2015/. Acesso em: 29 maio. 2019.

PAIM, Jairnilson Silva; ALMEIDA FILHO, Naomar de (org.). Saúde Coletiva: teoria e prática. 1.ed. Rio de Janeiro: MedBook, 2014, 702 p.

PESSÔA, Vera Lúcia Salazar; RUCKERT, Aldomar Arnaldo; RAMIRES, Julio Cesar de Lima (orgs.). Pesquisa qualitativa: aplicações em Geografia. Porto Alegre: Imprensa Livre, 2017. 568 p.

POLIDORO, Maurício; TAKEDA, Mariane Mayumi Garcia; BARROS, Omar Neto Fernandes. Mapeamento do índice de carência habitacional na Região Metropolitana de Londrina-PR. Geografia (Londrina), v. 18, n. 2, p. 75-87, 2009.

RIPSA, Rede Integrada de Informações para a Saúde. Indicadores básicos para a saúde no Brasil: conceitos e aplicações. 2. ed. Brasília: Organização Pan-americana da Saúde, 2008. Disponível em: http://tabnet.datasus.gov.br/tabdata/livroidb/2ed/indicadores.pdf. Acesso em: 10 mar. 2019.

SANTANA, P. Ambientes e sujeitos sociais no mundo globalizado: a geografia da saúde e as demais áreas do conhecimento. In.: OLIVEIRA, José Aldemir de; SOUZA, Geraldo Alves de (orgs.).

DOI:http://dx.doi.org/10.14393/Hygeia16049171 $\quad$ Hygeia $\quad$ v.16 $\quad$ p.25 - 36, 2020 página 35


Geografia da Saúde: ambientes e sujeitos sociais no mundo globalizado. Manaus: EDUA, 2016, p. 308.

SANTIAGO, Alynne da Costa; et al. Indicadores sociais e de saúde para a operacionalização da Vigilância à Saúde. Revista da Escola de Enfermagem da USP, v. 42, n. 4, p. 798-803, 2008. https://doi.org/10.1590/S0080-62342008000400025

SOUZA, Carlos Dornels Freire; COSTA, Klynger Farias da; RAMOS, Lucas da Silva. Distribuição Espacial das Intoxicações Exógenas por Agrotóxicos em Trabalhadores Rurais no Estado da Bahia Brasil, de 2007 a 2011. Hygeia - Revista Brasileira de Geografia Médica e da Saúde, v. 12, n. 23, p. $133-141,16$ dez. 2016. 